\title{
UN NUEVO MODELO DE RECLUTAMIENTO Y SELECCIÓN BASADO EN COMPETENCIAS: LA BOLSA UNIVERSITARIA DE TRABAJO U.N.A.M.
}

\author{
Laura Patricia Montoya Jiménez*
}

\section{Resumen}

En esta ponencia se abordan algunas de las dificultades que presentaban estudiantes y egresados de la UNAM que utilizaban el servicio de la Bolsa Universitaria de Trabajo (BUT) para insertarse en el mercado laboral de su profesión hasta el ciclo 1997-1998. Se explican los fundamentos y características de la reingeniería de procesos que dio origen al nuevo modelo de reclutamiento y selección basado en competencias de la BUT, destacando que la esencia del mismo es que no existen personas incompetentes, el reto de los procesos de reclutamiento y selección está en encontrar a la persona idónea para el puesto correcto, a partir de la evidencia de sus competencias.

Después de siete años de aplicación del modelo, se ha observado una mejora sustancial tanto en los servicios que brinda la BUT como en la calidad de la colocación de los candidatos universitarios en el mercado de trabajo. Finalmente, se hacen recomendaciones para dar respuesta a los retos que actualmente enfrenta.

\section{Abstract}

This exposition focuses on some of the difficulties faced by students and graduates of the UNAM who used the University Job Bank service to search for employment in their given field up to the academic year 1997-1998. This work also explains the foundations and characteristics of the process reengineering which gave rise to the new model of recruitment and selection based on BUT competencies, in turn notable for its essential notion that there are no incompetent candidates and that the challenge of the recruitment and selection process is finding the ideal candidate for the position, beginning with the evidence of its competencies.

Seven years after the model was applied, a substantial improvement in BUT services has been observed such as candidate placement quality in job market. Finally, several recommendations are offered in response to current challenges.

* Licenciada en Sociología, UNAM. Académica de la Dirección General de Orientación y Servicios Educativos; Instructora de los talleres que se imparten a estudiantes y egresados de la UNAM para apoyar su proceso de búsqueda de 
empleo y ha participado en diversos proyectos de investigación del Instituto Nacional de Salud Pública sobre el Mercado de Trabajo de los profesionistas de la salud.Imontoya@servidor.unam.mx

PALABRAS CLAVE: Competencias, bolsa de trabajo, ingreso mercado laboral, U.N.A.M.

\section{Introducción}

La Dirección General de Orientación y Servicios Educativos (DGOSE) de la UNAM, heredera de una tradición de más de 40 años de Orientación E-ducativa en la Institución, ha considerado con especial atención brindar apoyos y herramientas modernas que permitan a estudiantes y egresados insertarse con éxito en el mercado laboral de su profesión.

Hasta hoy, la inserción del estudiante y egresado de la UNAM en el mercado laboral sigue siendo la punta de lanza que preocupa no sólo a quien decide estudiar una carrera dentro de ella, sino también a las autoridades en general y en particular a quienes tienen en sus manos esta tarea.

Problemática1997-1998

En las reuniones del Grupo de Responsables de Bolsa de Trabajo de Fa-cultades y Escuelas, se manifestó que entre algunas de las dificultades que presentan los egresados que utilizan este servicio se tienen:

-Dificultad para identificar sus competencias y elaborar el currículum.

- No cuentan con suficientes habilidades para desempeñarse con éxito

durante una entrevista y son poco asertivos.

-Toman decisiones inmediatas, sin reflexionar sobre las condiciones

de contratación y funciones de puesto.

-Se muestran inseguros de sí mismos y de la formación alcanzada.

Datos consultados en 1997-1998 indicaban que por cada 3 egresados de educación superior, el país contaba con sólo un empleo. El $44 \%$ de los egresados de la UNAM se emplean a través de ofertas públicas y de ellos el $51 \%$ no satisface sus expectativas laborales originales 1.

Del total de carreras que se impartían en la UNAM hasta 1998 (68), el 20\% son de muy baja o nula demanda en el mercado de trabajo tradicional. 
Las causas pudieran ser:

-Una visión estrecha del mercado laboral.

-Las ofertas no consideran la diversidad de los perfiles profesionales del egresado universitario.

- Un porcentaje importante de empleadores conoce de forma limitada

el campo ocupacional de las carreras que se imparten en la UNAM.

-Los propios alumnos se autolimitan en la búsqueda de empleo, concentrándose únicamente en ofertas de su propia carrera.

Debido a la magnitud del problema, se integró una red conformada por los servicios de bolsa de trabajo de la UNAM que, con el intercambio de experiencias exitosas y el uso de la computación y las telecomunicaciones, sirviera de enlace entre la universidad y las instituciones oferentes de empleo en la magnitud del potencial de la Institución.

A partir de los problemas antes mencionados, la DGOSE se dio a la tarea de rediseñar el proceso de atención del servicio de la Bolsa Universitaria de Trabajo (BUT), para la búsqueda e instrumentación de un nuevo mo-delo que diera respuesta a las necesidades de los estudiantes y egresados de los diferentes niveles que ofrece la UNAM.

El propósito de este trabajo es presentar el modelo de competencias de la Bolsa Universitaria de Trabajo UNAM como una experiencia de replanteamiento y revaloración de la vinculación de la Universidad Nacional Autónoma de México con la sociedad y el sector productivo.

Desarrollodelmodelo

El proceso de reingeniería llevado a cabo para el desarrollo del modelo dio los siguientes pasos:

1. Análisis del proceso anterior de la Bolsa Universitaria de Trabajo.

2. Identificación de puestos y carreras de mayor demanda en las ofertas y vacantes de las empresas.

3. Definición de competencias generales con base en los requerimientos de los puestos. 
4. Panel de expertos para la validación de las competencias con gente responsable de la Dirección de recursos humanos o reclutamiento de personal.

De acuerdo con los datos obtenidos de la revisión del proceso de la Bolsa Universitaria de Trabajo, el sistema de atención estaba sustentado fundamentalmente en un servicio de información no automatizado de las ofertas y vacantes, la capacitación de algunos usuarios mediante el taller "Desarrollo de Potencial para la Búsqueda de Empleo" y la realización de entrevistas para puestos de nivel de mando. Este sistema alcanzaba índices de colocación de un $7 \%$ como promedio.

Con base en los resultados obtenidos del análisis de los datos estadísticos de la BUT en el período julio 1997-julio 1998, se identificaron algunos aspectos que vale la pena destacar:

- El $51 \%$ de los usuarios de la BUT fueron mujeres.

- En el $85 \%$ la edad osciló entre los 20 y los 35 años.

- En el $75 \%$ el campus de procedencia fue Ciudad Universitaria.

- La BUT logró colocar a egresados de 36 carreras (51\%) de las 68 ca-

rreras que se imparten en la UNAM.

- Los puestos de mayor demanda sólo consideraban cinco de estas

carreras.

Por otro lado, se identificó un mercado laboral que desconoce las potencialidades reales del egresado universitario y sus ámbitos de competencia, además de que los servicios profesionales no son solicitados en la proporción esperada.

Según los resultados de estos estudios, la DGOSE se dio a la tarea de rediseñar el proceso de atención del servicio de la BUT con el apoyo de un asesor externo; y se decidió instrumentar un nuevo modelo con el enfoque de competencias para estudiantes y egresados de los diferentes niveles que ofrece la UNAM.

Este nuevo modelo tiene su fundamento en el enfoque basado en competencias de Development Dimensions International (DDI), organización que durante más de 40 años ha trabajado en el estudio de las competencias y en cómo éstas se han usado, integrándolas como parte esencial de los sistemas de Recursos Humanos2. 
De acuerdo con este enfoque, las compañías están en busca de candidatos con perfiles flexibles, con habilidades que les permitan ge-nerar ideas novedosas, resolver problemas y asumir nuevos retos que coincidan con la visión y valores de las organizaciones. Por lo anterior, entonces, los procesos de reclutamiento y selección de personal buscan identificar los factores que inciden en el desempeño exitoso de los individuos que contratan, es decir sus competencias.

Existen diversas formas de definir las competencias. Las organizaciones: retoman su misión, visión, valores y objetivos, es decir su razón de ser; las personas lo relacionan con el nivel de éxito o logro que han alcanzado (estudiante de licenciatura, médico, abogado, doctor, etc.)

y a partir del puesto de trabajo, es decir, lo que hace que una persona tenga un desempeño exitoso.

El enfoque de nuestro modelo se centra en un acercamiento que considera cualquier comportamiento, conocimiento o motivación relevante en el trabajo como medida de lo que a un candidato le gusta o disgusta en el puesto.

En este sentido, este enfoque busca de cada candidato evidencias sobre su:

- Comportamiento relacionado con el puesto (cómo actúa).

- Conocimiento relacionado con el puesto (lo que conoce).

- Motivación relacionada con el puesto (lo que le gusta o disgusta).

Con base en lo anterior, ser competente en un trabajo está asociado a algo, competencias, que un candidato posee para desempeñarse con éxito en un puesto determinado.

Nuestro modelo consideró los siguientes supuestos:

- En cada puesto algunas personas se desempeñan en forma más efectiva que otras.

- Las más eficientes realizan actividades en forma específica y poseen características diferentes a las demás.

- Tales diferencias son apreciables y se pueden evaluar en resultados del puesto. 
Estos supuestos implican el concepto de que no existen personas incompetentes, es decir, que existe un puesto para cada persona y una persona para cada puesto, lo importante es lograr la conjunción de expectativas entre el trabajo y la persona.

Lo anterior llevó a la tarea de conocer cómo actúa, qué sabe y qué motiva a un egresado universitario para tener un desempeño sobresaliente en un puesto determinado, es decir, para este modelo el término competencias se refiere al conjunto de conocimientos, habilidades, comportamientos y motivaciones que tienen una correlación con el desempeño sobresaliente de los individuos en un puesto determinado.

Para poder validar el nuevo modelo del servicio de Bolsa de Trabajo e identificar las competencias que demanda el mercado laboral, se seleccionaron veintiuno del Directorio de Competencias y sus Acciones Clave de Celanese Mexicana, S.A. de C.V. y se integró un Grupo de Enfoque con la participación de treinta y cuatro responsables del área de recursos humanos y selección de personal de los niveles directivos y gerenciales de treinta y dos empresas de los sectores público, social y privado que más demandan egresados de la UNAM.

Las competencias que permitirían correlacionar las que poseen los universitarios con las que el trabajo requiere, estuvieron determinadas por las características del puesto de acuerdo con:

- Nivel: derivadas de la categoría a la que pertenece el puesto (director, gerente, superintendente, jefe de departamento, asistente, espcialista, profesor, etc.).

- Específicas del puesto: en función de las cualidades que se requieren para desempeñar un trabajo específico (Gerente de Comunicaciones, Director de Carrera, Superintendente de Producción, Profe-

sor de Matemáticas, Analista Contable, etc.).

Los resultados obtenidos de esta primera reunión, sustentaron el desarrollo de guías de entrevista para las veintiún competencias esperadas para los catorce puestos más demandados. Estas competencias fueron señaladas por el Grupo de Enfoque en los siguientes porcentajes:

Habilidad para Persuadir/Vender 57.78

Conocimientos Técnico/Profesionales 50.69

Planeación y Organización 47.78 
Impacto 46.30

Adaptabilidad 41.67

Generar Confianza 40.97

Tolerancia al Stress 40.74

Aprendizaje Continuo 40.00

Toma de Decisiones 39.68

Tenacidad 38.89

Iniciar Acciones 37.96

Orientación al Cliente 37.22

Innovar 35.00

Presentación Formal 33.33

Trabajo en Equipo y Colaboración 31.48

Asesoría 29.17

Manejar los Conflictos 27.78

Control de la Información 20.83

Negociación 20.83Xihmai 29

Posteriormente los responsables de recursos humanos y selección de personal determinaron, a través de una consulta individual, por consenso, las cinco competencias básicas que todo universitario debía tener para ingresar a cualquier puesto de trabajo a nivel profesional:

Adaptabilidad

Toma de decisiones

Iniciativa

Aprendizaje continuo

Comunicación 
Cabe mencionar que cada competencia señalada fue definida por el grupo y se determinaron preguntas de comportamiento y acciones clave para evidenciarlas, lo cual posibilitó la estructuración de las guías de entrevista.

Por ejemplo, para rastrear la competencia Aprendizaje Continuo tenemos:

- Definición: identificar activamente nuevas ideas para fomentar el aprendizaje.

- Acción clave: interesarse en actividades de aprendizaje o maximizar el aprendizaje.

- Pregunta de comportamiento clave: en cualquier nuevo empleo, hay cosas que sabemos y otras que requieren aprendizaje, platíqueme ¿qué hace para obtener este conocimiento?

De esta forma, los elementos que integran nuestro modelo de atención basado en competencias son:

- Competencias generales requeridas por las empresas, de acuerdo con estrategias presentes y futuras.

- Competencias correspondientes al puesto a contratar.

- Competencias de los alumnos y egresados de la UNAM.

En la práctica, la correlación de competencias está determinada por un sistema de "Selección Eficaz" basado en entrevistas para la identificación de competencias. La Selección Eficaz consiste en un proceso organizado en el que, mediante una serie de entrevistas, con personas diferentes, se obtiene información sobre las competencias de los candidatos a partir de evidencias acerca de comportamientos pasados para predecir futuros, asegurando la imparcialidad, efectividad y aceptación sobre la toma de decisiones de selección de candidatos3 .

Como producto de esta reingeniería, la (BUT) ofrece hoy día un servicio promovido por la Secretaría de Servicios a la Comunidad de la UNAM, a través de la DGOSE cuyo objetivo primordial es:

Apoyar al estudiante y egresado de la UNAM en su incorporación al mercado de trabajo dotándole, bajo un modelo de competencias, de las estrategias y herramientas que le permitan satisfacer sus expectativas y motivaciones, enriqueciendo así su relación con el mercado laboral. 
Este objetivo se pretende lograr por medio de tres estrategias principales:

- Sistema de Selección Eficaz: consiste en una preselección basada en tres entrevistas estructuradas para identificar competencias.

- Apoyo a los alumnos y egresados para identificar sus competencias, elaborar su currículum y preparar su entrevista a través de un taller de seis horas de duración: Obteniendo el Trabajo que Deseo, con el correspondiente manual que le sirve al usuario como un instrumento permanente.

- Realizar una preselección acertada de los candidatos que se envían a las empresas oferentes de empleo.

Aplicación y resultados del modelo

A siete años de operación del modelo basado en competencias de la (BUT), la respuesta y participación de los responsables de bolsa de trabajo de facultades y escuelas ha sido comprometida y entusiasta, lo que sentó las bases para:

Establecer un Sistema Universitario de Bolsa de Trabajo que permitió unificar criterios en el quehacer de las áreas que brindan el servicio en las facultades y escuelas

-4 escuelas

-13 facultades

- 5 facultades de estudios superiores

- 3 direcciones generales.Xihmai 3

Extender el modelo basado en competencias en toda la Universidad.

- Taller Formación de Instructores (2 veces al año)

Contar con un sistema automatizado para el registro y consulta de ofertas de empleo, así como para consulta de candidatos UNAM. 


\begin{tabular}{|l|c|c|c|c|c|}
\hline $\begin{array}{l}\text { Vacantes } \\
\text { registradas }\end{array}$ & $\mathbf{2 0 0 3}$ & $\mathbf{2 0 0 4}$ & $\mathbf{2 0 0 5}$ & $\mathbf{2 0 0 6}$ & $\mathbf{2 0 0 7}$ \\
\hline TOTAL & 7,664 & 12,930 & 17,456 & 12,544 & 27,304 \\
\hline
\end{tabular}

Tabla 1. Vacantes Registradas

Fortalecer y diversificar los vínculos con el sector productivo.

\begin{tabular}{|l|c|c|c|c|c|}
\hline $\begin{array}{l}\text { Empresas } \\
\text { registradas }\end{array}$ & $\mathbf{2 0 0 3}$ & $\mathbf{2 0 0 4}$ & $\mathbf{2 0 0 5}$ & $\mathbf{2 0 0 6}$ & $\mathbf{2 0 0 7}$ \\
\hline TOTAL & 1,209 & 1,524 & 1,941 & 2,287 & 2,774 \\
\hline
\end{tabular}

Tabla 2. Empresas Registradas

Ajustar el modelo a una entrevista de preselección, toda vez que la realización de tres entrevistas resultó inoperante al incrementarse la demanda de las empresas y universitarios.

Diversificar las opciones de capacitación para estudiantes y egresados durante su proceso de búsqueda de empleo.

Taller Obteniendo el Trabajo que deseo

6 hrs.

1999

Taller Elaboración del Currículum

3 hrs.

2003

Taller Preparando mi Entrevista de Trabajo

$3 \mathrm{hrs}$.

2003

Incrementar y diversificar la demanda de carreras y escuelas de procedencia de los universitarios. 


\begin{tabular}{|l|c|c|c|c|c|}
\hline $\begin{array}{l}\text { Carreras } \\
\text { demandadas }\end{array}$ & $\mathbf{2 0 0 3}$ & $\mathbf{2 0 0 4}$ & $\mathbf{2 0 0 5}$ & $\mathbf{2 0 0 6}$ & $\mathbf{2 0 0 7}$ \\
\hline TOTAL & 52 & 56 & 61 & 75 & 73 \\
\hline
\end{tabular}

Tabla 3. Carreras demandadas

Organizar siete ediciones de la Feria del Empleo UNAM en la que participan todas las facultades y escuelas.

\begin{tabular}{|c|c|c|c|}
\hline AÑO & $\begin{array}{c}\text { NÚMERO } \\
\text { EMPRESAS }\end{array}$ & $\begin{array}{c}\text { NÚMERO } \\
\text { VACANTES }\end{array}$ & $\begin{array}{c}\% \\
\text { OCUPACIÓN } \\
\text { VACANTES }\end{array}$ \\
\hline $\mathbf{2 0 0 1}$ & 110 & 2,450 & 89.7 \\
\hline $\mathbf{2 0 0 2}$ & 126 & 2,500 & 90.1 \\
\hline $\mathbf{2 0 0 3}$ & 145 & 2,920 & 88.9 \\
\hline $\mathbf{2 0 0 4}$ & 200 & 3,215 & 91.0 \\
\hline $\mathbf{2 0 0 5}$ & 220 & 5,300 & 91.0 \\
\hline $\mathbf{2 0 0 6}$ & 234 & $6.350^{*}$ & 90.3 \\
\hline $\mathbf{2 0 0 7}$ & 245 & 7,054 & En proceso \\
\hline
\end{tabular}

Tabla 4. Ferias del Empleo UNAM

Tabla 3. Carreras demandadas

Organizar siete ediciones de la Feria del Empleo UNAM en la que participan todas las facultades escuelas.AÑO2001200220032004200520062007NÚMEROEMPRESASNÚMERO VACANTES\%OCUPACIÓNVACANTES1101261452002202342452,4502,5002,92 03,2155,3006.350*7,05489.790.188.991.091.090.3En proceso

Tabla 4. Ferias del Empleo UNAM

Definir lineamientos generales para atender programas de prácticas profesionales, becas trabajo, trainees, simuladores de negocios, entre otros.

Kraft, HSBC, Unilever, Procter \& Gamble, GNP, L'Oreal, Abengoa, Maerks, Phillip Morris, Nestlé, Colgate, 3M de México, Gigante, Gobierno del Distrito Federal, Danone.

Realizar reclutamientos especiales, a solicitud de empresas e instituciones, con participación de estudiantes y egresados de diversas carreras. 
Procter \& Gamble, Kraft, INROADS, Ford Motor Company, HSBC, Secretaría de Seguridad Pública, Universidad Autónoma de Ciudad Juárez, Maerks México, Cargill, Halliburton, Paradigm, Progresemos, LG Electronics Monterrey, Abengoa, L'Oréal, Management Solutions, Pepsico, Xihmai 33

Grupo Fidalex, GNP, Unilever, Universidad del Valle de México.

Conformar una red que ha facilitado el intercambio de información y experiencias.

Reuniones mensuales del Grupo de Responsables de la Bolsa de Trabajo de Facultades y Escuelas UNAM (primer martes de cada mes).

Diversificar las líneas de trabajo académicas al interior de la BUT.

Incrementar la cantidad y calidad de colocación de los alumnos y egresados de la institución.

\begin{tabular}{|c|c|c|c|c|c|}
\hline Colocación & $\mathbf{2 0 0 3}$ & $\mathbf{2 0 0 4}$ & $\mathbf{2 0 0 5}$ & $\mathbf{2 0 0 6}$ & $\mathbf{2 0 0 7}$ \\
\hline$\%$ & 52 & 64 & 80 & 81 & 82 \\
\hline
\end{tabular}

Tabla 5. Porcentaje de Colocación

Conclusiones y recomendaciones

Las bondades en la aplicación de este modelo se han visto reflejadas en un avance cualitativo y cuantitativo de los servicios que la BUT ofrece a los universitarios, empresas e instituciones públicas, lo cual ha quedado evidenciado en los diversos procesos de evaluación que la BUT realiza con estos actores. No obstante se enfrentan nuevos retos, para lo que proponemos las siguientes acciones:

Organizar el Segundo Grupo de Enfoque para validar el funcionamiento y hacer modificaciones al Modelo Basado en Competencias.

Desarrollar la segunda fase del sistema automatizado con que cuenta la BUT. 
Mejorar los mecanismos de seguimiento de candidatos a empleo que son atendidos en la BUT.34 Xihmai 


\section{FuentesdeConsulta}

BYHAM William C, 1997, Landing the job you want, Pittsburgh, PA: Development Dimensions International.

----, 1995, The Selection Solution, Pittsburgh, PA: Development Dimensions International.

----, 1996, Competencies and Organizational Success, Pittsburgh, PA: Development Dimensions International.

Celanese Mexicana S.A. de C.V. Directorio de Competencias y sus Acciones Clave. Development Dimensions International. 1998.

DEVELOPMENT DIMENSIONS INTERNATIONAL. Competencies. Charting Course for success.1997. DEVELOPMENT DIMENSIONS INTERNATIONAL. Targeted Selection: A Behavioral approach to improved hiring decisions. 1989.

----, Developing Dimension Competency based Human Resource System. 1996.

----, Chartings Course for Success. How to put competencies to work in your organization, 1996.

----, Presentación División Latinoamérica, 1997.

GómezD. Javier H. Manual para el Taller "Obteniendo el Trabajo que Deseo". DGOSE.UNAM 1998.

---, Manual para el Curso de Formación de Instructores para el Taller "Obteniendo el Trabajo que Deseo". DGOSE.UNAM 1998.

MUÑOZ Izquierdo, Carlos, Diferenciación Institucional de la Educación Superior y Mercado de Trabajo, Colección ANUIES 1998. 